\title{
ENTRE EL GAJO Y LA LENGUA: BERENGUER Y LOS LÍMITES DEL SUJETO
}

POR

SEBASTIÁN URLI

University of Pittsburgh

Université de Paris 8

A María Lucía Puppo, Por Amanda, por la confianza, por indicarme la constelación

Quisiera partir de una afirmación, de una constatación: hay en la poesía de Amanda Berenguer un predominio del sujeto. Esto no implica restarle importancia a las reflexiones sobre el lenguaje, el lugar o la temporalidad con que convive dicho sujeto sino aceptar que esos otros elementos, que esas otras coordenadas que enmarcan su existencia y todo acto de enunciación deben leerse, en última instancia, desde y frente a ese predominio. Sin embargo, las líneas que conforman el mapa de dicha preminencia son múltiples y variadas y cambian según el poemario o poema en cuestión, como así también la noción de sujeto que puede entenderse, en un primer momento, como aquel que enuncia, esto es, como un deíctico pero también en un sentido gramatical "tradicional" como aquel de quien se predica algo y aquel que realiza o padece un número de acciones en ciertas circusntancias. De este modo, el predomino del sujeto no debe entenderse necesariamente como el de un yo lírico, aunque este, como veremos, cumple en muchos poemas un papel central, sino más bien en un sentido amplio que permite aceptar que una tercera persona también es sujeto $\mathrm{y}$, al mismo tiempo, que sujeto puede leerse, muchas veces, en relación con sujeción, tanto como aquello que sujeta como también aquello que es sujetado. Quisiera entonces agregar una segunda afirmación que completa y matiza la primera: hay en la poesía de Berenguer, quizás como un gesto de sospecha ante el predominio mencionado, quizás como un horizonte que lo enmarca y le da cabida, una experimentación constante con los límites del sujeto enunciante (en muchos casos explícitamente femenino) y con el lenguaje con el que este (se) configura.

Uno de estos límites es, sin duda, la propia lengua poética y las reflexiones en torno a ella que son una constante en su poesía (y quizás en la de todo poeta) y que aparecen en diversos poemarios. Pero existen otros límites más precisos como la postulación o 
la búsqueda de un lugar de enunciación tensionado entre un adentro y un afuera que no parece resolverse nunca, como ocurre en La botella verde (1995); la desaparición de personas producto de la violencia política, ausencia que es al mismo tiempo una presencia constante y que en su calidad de ausencia siempre presente o, si se quiere, de presencia siempre ausente, reconfigura al sujeto que enuncia, como ocurre en Los signos sobre la mesa (1988); o el uso de la fábula didáctica "infantil" o de enseñanza escolar para extrañar el lenguaje y convertirlo en una especie de vecindario por el que el sujeto puede deambular y reflexionar sobre la materia con la que trabaja y sobre la identidad, tal como ocurre en Casas donde viven criaturas del lenguaje (No se sienta Usted loco, es arquitectura para pocos) y El diccionario (2005), por citar solo algunos casos paradigmáticos.

Ahora bien, existe otro límite, que es el que me interesa explorar en este ensayo, y es justamente aquel que inaugura, que posibilita, la presencia de objetos o cosas que el sujeto percibe, que el sujeto nombra, toca, explora y frente a la cual, a la par que se proyecta y se configura, encuentra un límite a dicho proceso de figuración. Me refiero, aunque el lector o la conocedora de la obra de Berenguer ya lo habrá adivinado, a la presencia de las manzanas, las naranjas, las uvas, etc. en el poemario Identidad de ciertas frutas de 1983.

En él encontramos veintinueve poemas dedicados, obviamente, a diversas frutas. En algunos casos se trata de una descripción del objeto a partir de comparaciones o recursos literarios que destacan aspectos que de otro modo pasarían desapercibidos. En otros, y el breve inventario descriptivo que propongo aquí no supone que una fruta no pueda estar en más de un tipo de caso, se pone en primer plano la relación del sujeto lírico que enuncia con la fruta en cuestión y las sensaciones que esta le provocan o provocaron al desgustarla. En otros varios, la descripción de la relación entre el sujeto y el objeto no se limita a las sensaciones, en su mayoría visuales, táctiles y gustativas que posibilita la relación (el saborear la fruta), sino que la presencia de la fruta se conecta con algún recuerdo o momento personal del sujeto, se expande en una evocación del pasado o de ciertos deseos o frustraciones que operan más allá de la captación sensorial del objeto en cuestión. Y en un último caso (aunque puede haber otros), encontramos ciertos momentos en los que lo que parecería primar es la alteridad de la fruta en tanto fruta y la imposibilidad de conocerla completamente. Sin embargo, lo que creo que acontece en esta dialética entre el sujeto y el objeto es, para retomar las categorías heideggerianas de tierra y mundo, una constante oscilación entre aquello que la fruta y el sujeto posibilitan (el mundo que proyectan) y aquello que no revelan, aquello que ocultan (la tierra). Cómo señala Heidegger en su conocido ensayo:

El mundo es la abierta apertura de las amplias vías de las decisiones simples y esenciales en el destino de un pueblo histórico. La tierra es la aparición, no obligada, de lo que siempre se cierra a sí mismo y por lo tanto acoge dentro de sí. Mundo y tierra son

Revista Iberoamericana, Vol. LXXXII, Núm. 257, Octubre-Diciembre 2016, 135-153 ISSN 0034-9631 (Impreso)

ISSN 2154-4794 (Electrónico) 
esencialmente diferentes entre sí y, sin embargo, nunca están separados. El mundo se funda sobre la tierra y la tierra se alza por medio del mundo. Pero la relación entre el mundo y la tierra no va a morir de ningún modo en la vacía unidad de opuestos que no tienen nada que ver entre sí. Reposando sobre la tierra, el mundo aspira a estar por encima de ella. En tanto que eso que se abre, el mundo no tolera nada cerrado, pero por su parte, en tanto que aquella que acoge y refugia, la tierra tiende a englobar al mundo y a introducirlo en su seno. (34-35) ${ }^{1}$

Ahora bien, no es cuestión de limitarse a decir que en el poemario de Berenguer uno de los dos polos de la relación, el sujeto o la fruta, se corresponde unívocamente con el mundo o la tierra, con la proyección o con el ocultamiento (de hecho en Heidegger el templo griego en tanto mundo que se proyecta y la tierra donde se erige no se pueden separar completamente), sino que lo me interesa apuntar es que tanto el sujeto que percibe o enuncia así como también la fruta (que, y hay que decirlo, es a veces el sujeto gramatical de un verso o una oración) proyectan un mundo, producen un mundo y un cambio en el otro pero, al mismo tiempo, ocultan algo, no pueden ser subsumidos en ese otro que se les impone como un límite en la relación.

Si propuse un predominio del sujeto que enuncia y percibe sobre la fruta (predominio fundado en un cuestionamiento constante de la preminencia y de los límites del sujeto) no es porque ésta quede subsumida al sujeto, sino más bien porque no encontramos

\footnotetext{
Y agrega Heidegger a continuación. "Este enfrentamiento entre el mundo y la tierra es un combate. Confundimos con demasiada ligereza la esencia del combate asimilándolo a la discordia y la riña y por lo tanto entendiéndolo únicamente como trastorno y destrucción. Sin embargo, en el combate esencial, los elementos en lucha se elevan mutuamente en la autoafirmación de su esencia. La autoafirmación de la esencia no consiste nunca en afirmarse en un estado casual, sino en abandonarse en el oculto estado originario de la procedencia del propio ser. En el combate, cada uno lleva al otro por encima de sí mismo. De este modo, el combate se torna cada vez más combativo, más propiamente eso que verdaderamente es. Cuanto más duramente se supera a sí mismo y por sí, tanto más implacablemente se abandonan los contendientes a la intimidad de un simple pertenecerse a sí mismo. Para aparecer ella misma como tierra en el libre aflujo de su cerrarse a sí misma, la tierra no puede prescindir de lo abierto del mundo. Por su parte, el mundo tampoco puede deshacerse de la tierra sí es que tiene que fundarse sobre algo decidido como reinante amplitud y vía de todo destino esencial. Desde el momento en que la obra levanta un mundo y trae aquí la tierra, se convierte en la instigadora de ese combate. Pero esto no sucede para que la obra reduzca y apague de inmediato la lucha por medio de un insípido acuerdo, sino para que la lucha siga siendo lucha. Al levantar un mundo y traer aquí la tierra, la obra enciende esa lucha. El ser-obra de la obra consiste en la disputa del combate entre el mundo y la tierra. Es precisamente porque la lucha llega a su punto culminante en la simplicidad de la intimidad por lo que la unidad de la obra ocurre en la disputa del combate. La disputa del combate consiste en agrupar la movilidad de la obra, que se supera constantemente a sí misma. Por eso, es en la intimidad del combate donde tiene su esencia el reposo de la obra que reposa en sí misma" (35). En nuestro caso, y a modo de analogía, podría indicarse que el ser sujeto del sujeto y el ser objeto del objeto también se funda en esa tensión entre la relación y el mundo que cada uno de los participantes de la relación inaugura y aquello que no se devela, aquello que no cede y que en tanto límite configura y (des)delimita a los participantes.
}

Revista Iberoamericana, Vol. LXXXII, Núm. 257, Octubre-Diciembre 2016, 135-153 ISSN 0034-9631 (Impreso) ISSN 2154-4794 (Electrónico) 
poemas en los que el objeto enuncie desde un yo fruta (en una especie de prosopopeya). Por el contrario, los poemas presentan relaciones dinámicas entre sujeto y objeto (aunque desde la perspectiva de un sujeto no-fruta), personificaciones en tercera persona o, a veces, y con eso comenzaré el análisis, poemas donde no encontramos ni un solo deíctico que asuma la enunciación ni un sujeto gramatical del cual puedan predicarse atributos o acciones (y si se predica algo lo que se predica es cierta pasividad). Pero este predominio del sujeto, fundado, insisto, en el cuestionamiento constante de su preeminencia, motivado por el cuestionamiento de dicha preeminencia, no implica que el sujeto o el objeto se pierdan por completo en la relación o sean completamente modificados hasta el punto de desaparecer el uno en el otro, sino que hay algo, tanto en las modificaciones que padece la fruta al ser saboreada como el sujeto humano al verse afectado por la presencia irreductible de la fruta y su poder de evocación de recuerdos, que permanece oculto y no se revela, tanto en el caso del sujeto que no desea proyectarlo o no logra proyectarlo en la fruta como en ésta que se niega a entregarse a una ontología completamente relacional aun cuando sea devorada.

Comenzaré el ensayo por el segundo poema del libro para desde él proponer un recorrido que conjugue el análisis de ciertos poemas o ciertos versos sintomáticos con algunas reflexiones de otros escritos de Berenguer y de algunos aportes de la crítica sobre poesía. El poema en cuestión, el segundo del libro, se titula "(la manzana II)" y en el leemos lo siguiente:

\author{
Una manzana color manzana \\ otra manzana sin cáscara \\ color de otra manzana \\ otra manzana desaparecida \\ saboreada: \\ de las tres ¿cuál la manzana verdadera? (417)
}

Lo primero que quisiera señalar es que es muy difícil, si no imposible, postular, dentro del poema como acto lingüístico, un sujeto. No hay ningún deíctico de ninguna clase pero tampoco tenemos ningún verbo que pueda indicar una acción particular predicable de un sujeto o que afecte a un sujeto o que muestre en su terminación verbal gramatical la posibilidad de un sujeto. Se podría pensar que las manzanas están y que ese estar implica ya una acción pero tampoco tenemos una superficie o un lugar donde las manzanas pueda decirse que están. ¿Se trata de una mesa? ¿Se trata de la mirada de aquel, que no se nombra, que percibe las manzanas? ¿Del poema y del lenguaje como instancias que imposibilitan toda subjetividad ${ }^{2}$ ¿De la mirada, pero ya entonces

2 Al respecto pueden servir estas palabras de Emilio Irigoyen en uno de los pocos trabajos sobre Berenguer (aunque en este caso escritas a partir de La botella verde): "En realidad creo que no es posible ceñirse

Revista Iberoamericana, Vol. LXXXII, Núm. 257, Octubre-Diciembre 2016, 135-153 ISSN 0034-9631 (Impreso) 
estaríamos por fuera del ámbito del enunciado poético, del lector? Sin duda que la del lector es una posibilidad. Pero quisiera tratar de pensar en este trabajo, al menos por el momento, en si es posbile hallar un sujeto en los versos y oraciones que conforman este poema en particular.

Se podría objetar que el participio "desaparecida", que como todo verboide posee una valencia doble (puesto que remite a una acción y a un sujeto u objeto al que modifica, en este caso como adjetivo) podría implicar la presencia de un sujeto. Sin embargo, nada garantiza que el "desaparecida" resuelva la posibilidad de definir si la manzana es un sujeto o un objeto. Hay otra potencial objeción a la que me anticipo, la de la posibilidad de un predicado no verbal en la pregunta que cierra el poema donde el verbo copulativo ser o algún otro funcionarían como acción y por lo tanto postularían un sujeto. Si bien esta objeción me parece plausible, el hecho de que no se coloque el verbo (en ese predicado no verbal) sumado a la ausencia de otros verbos y al hecho de que en caso de que aceptemos la presencia ausente del verbo en el predicado no verbal dicha presencia reimitiría a una duda que cuestiona la posibilidad misma de la presencia de la manzana, o al menos de una manzana, (puesto que el predicado no verbal aparece dentro de una pregunta y puede remitir o bien al cuál que es un pronomobre interrogativo que no define o a la manzana que está siendo cuestionada, no sabemos bien por quién o desde dónde en el poema), estos hechos, insisto, permiten plantear el problema desde otro lado, desde el rastro o el resto de una posibilidad de sujeto. En definitiva si la mazana está siendo cuestionada hay alguien o algo que la cuestiona, aun cuando no esté explicita esa presencia en el poema.

Asimismo, hay un detalle que permite vislumbrar o postular este resto (que personalmente identifico y quiero leer como un resto de sujeto aunque sólo se apreciará esta decisión al final del ensayo) en el "tres" que antecede a la pregunta final. En la elección de "tres" como resumen de las posibles manzanas hay ya una elección. De hecho, el poema presenta más de tres manzanas: creo contar cuatro aunque hay una quinta en ausencia 1. la manzana color manzana. 2. Esa manzana que otorga el color podría ser otra manzana. 3. Otra manzana, esta vez sin cáscara pero que tiene el color de una cuarta manzana que aparentemente es distinta a las otras. La quinta manzana sería la ausente, ya devorada, que el poema postula como diferente, por el hecho de que no se la coloca en relación al sustantivo "color" como una de las tres principales.

en este libro a un objeto que funcione como tema; a ningún objeto, en ningún momento. Es más, casi parece que no sólo no hay un objeto, sino que tampoco es posible ceñirse a un SUJETO [sic]. La propia voz que dice estos poemas, que cuenta cosas desde el interior de una botella, no funciona en el libro como la causa sino por el contrario como la consecuencia de estos poemas, su resultado, su remanente, o su pivote (depende del lugar del que se mire). Son los poemas los promotores de que haya un sujeto para esta escritura" (41).

Revista Iberoamericana, Vol. LXXXII, Núm. 257, Octubre-Diciembre 2016, 135-153 ISSN 0034-9631 (Impreso)

ISSN 2154-4794 (Electrónico) 
Por supuesto que contar manzanas, pesarlas y descubrir si se trata de medio kilo o de dos no nos sirve de mucho para lo que me propongo aquí que es más bien dar cuenta de la (con)figuración del sujeto que opera en el poemario de Berenguer. No obstante, ese "tres" implica si no la presencia al menos un resto de sujeto que no parece dispuesto a desaparecer y que ha decidido que se trata de tres manzans y que parece, por un lado, no poder subsitir ante la presencia de las manzanas que son el centro de la escena (lo único que parece haber), pero que, por el otro, parece distanciarse de ellas al elegir que se trate de tres y no de cuatro o cinco y al colocar "las tres" y no "nosotras tres" impiendo una identificación con la manzana, en tanto prosopopeya. De hecho, la posibilidad de que un lector pueda contar más o menos manzanas indica la presencia de algo o alguien que dentro del poema se constituye como diferente a la manzana o las manzanas. Esto, como queda claro, no permite postular una disolución del sujeto sino que aún en el linde con lo impersonal, podemos encontrar ciertos rastros. Como explica Culler en un trabajo a estas alturas clásico:

El personaje poético es una construcción, una función del lenguaje del poema, pero, aun así, desempeña el papel de unificador del sujeto individual, y hasta poemas que dificultan la construcción de un personaje poético se basan para sus efectos en que el lector haya de construir una situación enunciativa. Como sostiene Henri Meschonnic en un artículo acertado sobre Kristeva, es más fructífero subrayar la impersonalidad de la escritura y el significado producido por el intento de construir un personaje ficticio que hablar de la desaparición del sujeto. (La poética 242)

Esta necesidad de que el lector construya una posibilidad de sujeto dentro de ciertas coordenadas enunciativas no quisiera, sin embargo, que se confundiera con la imposibilidad de rastrear la presencia de un sujeto que enuncia "dentro" del poema. Que el lector construya una situación enunciativa puede entenderse, al menos, de dos modos distintos aunque no necesariamente excluyentes: por un lado, y en relación con la impersonalidad de los deícticos, el lector puede asumir la voz, asumir la posición del yo que enuncia, y ampliar la referencialidad del deíctico. Por otra parte, que el lector precise recrear la situación enuncitativa implica la importancia de dicha situación enunciativa y de la identificación, aunque sea parcial, de algunos elementos que la conforman (como el sujeto que enuncia) para de esa manera dotar de un posible significado aquello que se lee. En este caso, sin embargo, no encontramos ningún deíctico y la identificación de un posible sujeto se reduce, al menos en apariencia, a un resto. Exploremos un poco más esta idea del resto.

Para Noé Jitrik, el resto “es lo que queda después de que todo lo demás ha desaparecido, las palabras empleadas, el modo de la enunciación, la referencia, la estructura, el resto interno y la lucha por el espacio" (214). Aunque enseguida agrega: "Pero en el caso de la poesía se diría que el "resto" es una condición, no lo que ha

Revista Iberoamericana, Vol. LXXXII, Núm. 257, Octubre-Diciembre 2016, 135-153 ISSN 0034-9631 (Impreso) 
quedado, difícil de establecer, sino "lo que debe quedar", si el poema ha sido logrado, como lo irreductible de su presencia (215). En el caso del poema que he estado analizando, ese resto que debe quedar, ese resto "condición" se manifiesta, por un lado, y se trata de algo puntual, en esa no presencia presente del sujeto, no muy precisa en el poema, que reúne la multiplicidad de las manzanas en un "tres", que las delimita en un tres, aun cuando la pregunta del final tenga tintes irónicos. Pero ese resto también se manifiesta en la necesidad de presentar los límites del conocimiento de la fruta, algo que la misma pregunta irónica postula aun cuando la respuesta, si entendemos que se trata de una pregunta retórica, ya está dada en el poema: la multiplicidad (por ende no habría una identidad de la fruta diferente a la de las múltiples manifestaciones).

Ahora bien, podríamos leer la caracterización del resto que propone Jitrik de otro modo, al menos la caracterización de la primera parte de la cita. Así, entonces, el resto ya no sería lo que queda después de que todo ha desaparecido sino lo que desparece después de que todo se ha mostrado. O en otras palabras, lo que se oculta cuando parece que ya todo se ha mostrado, lo que permitiría postular otra forma de aproximación a la identidad de la fruta, una más interesada por aprehender aquello que no se muestra. Veamos un ejemplo concreto. El poema dedicado al damasco:

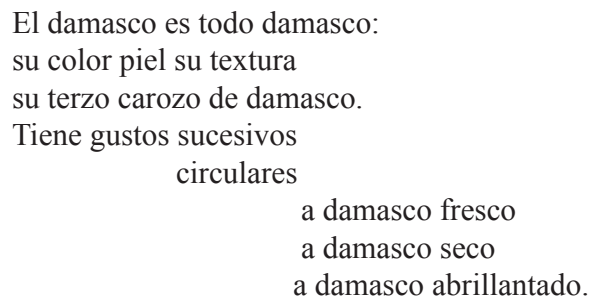

El árbol de damasco

se sueña a sí mismo. (451)

Aquí, si bien encontramos una delimitación parecida, un resto del sujeto delimitador, al del poema de la manzana, un resto que indica que hay tres damascos y que se aroga la potestad de usar un verbo como "tiene" (que implica cierta exterioridad respecto aquello que tiene, respecto al sujeto u objeto que tiene gustos sucesivos, en este caso el damasco), a diferencia del poema de la manzana encontramos que la fruta es sujeto gramatical de la oración (la manzana no lo era en el otro poema) y que la fruta parece bastarse a sí misma, de allí que el damasco sea todo damasco y que el árbol de damasco se sueñe a sí mismo. Hay un intento en el poema de postular lo que la fruta es sin necesidad de un sujeto que la perciba o de una relación con otros objetos pero, al mismo tiempo, en la cicularidad del poema, se vislumbra un intento de impedir que la fruta se muestre a un otro exterior en su total profundidad o ser.

Revista Iberoamericana, Vol. LXXXII, Núm. 257, Octubre-Diciembre 2016, $135-153$ ISSN 0034-9631 (Impreso) 
Por supuesto que, como vimos con el poema de la manzana, y de allá el interés y la complejidad del poemario de Berenguer, esto no siempre se logra y la relación con un otro aparece aunque no sea más que de un modo lateral. Después de todo desde dónde sino desde una relación se puede plantear la frescura o lo abrillantado o seco del durazno. Aun así, sin embargo, el poema intenta hablar y concebir la fruta desde una perspectiva diferente a la de una mera percepción o fenómeno mediado por un sujeto que percibe.

En este sentido me parecen oportunas algunas reflexiones de Graham Harman, que además basa parte de su análisis en un relectura de Heidegger, y también las de García desde la perspectiva de una filosofía orientada al objeto no porque esté de acuerdo con todos los puntos que proponen (de hecho he comenzado con la afirmación del predominio del sujeto y sigo dentro de la diferencia entre un sujeto humano y un objeto no humano) sino porque alertan, aun en la centralidad que le dan al objeto, sobre los riesgos de una concepción puramente relacional de la identidad aunque sin por eso llegar a negar la importancia de las relaciones en la conformación identitaria.

Según Harman "The only way to do justice to objects is to consider that their reality is free of all relation, deeper than all reciprocity. The object is a dark crystal veiled in a private vacuum: irreducible to its own pieces, and equally irreducible to its outward relations with other things" (The Quadruple Object 47). Harman contrasta entonces un objeto sensual (y quizás sería más apropiado pensarlo como una dimensión del objeto, aun cuando este no sea el vocabulario empleado por el autor) que está en otra cosa que en sí mismo (en el objeto que lo percibe) y el objeto real (que es en sí mismo). Harman explica, mediante el ejemplo del martillo que retoma de Heidegger, que el hecho de que un objeto pueda no funcionar tal como quisiera aquel que lo emplea es lo que permite dudar de aquellas posturas filosóficas que le niegan al objeto una existencia ajena al uso o a su manifestación ante la conciencia. Y esto lo vincula el filósofo estadounidense con la posibilidad del cambio. Si la mesa o la cama u otro objeto no tuvieran cierta existencia autónoma a cómo son percibidas por la consciencia no se podrían romper y las situaciones cambiar. Un perro que no percibo no podría aparecer por detrás y moderme y, sin embargo, esas cosas ocurren más allá del valor de uso que puede darle un sujeto o a las cualidades de un objeto que puedan manifestarse a una conciencia determinada en un espacio y tiempo determinados. En palabras del autor:

if there were no hidden hammer-in-itself, but only a series of determinate hammermanifestations, then no change could ever occur in the world. For every manifestation would already be all that it is, holding no surplus in reserve that could surge forth in a later moment and be expressed in the world. The fact that there is change in the world shows that something lies behind any manifestation (this is essentially the same argument as Heidegger's against Husserl) and is capable of manifesting differently under different circumstances. ("Tristan García and the Thing in-Itself" 6)

Revista Iberoamericana, Vol. LXXXII, Núm. 257, Octubre-Diciembre 2016, $135-153$ ISSN 0034-9631 (Impreso) 
Lo que Harman intenta evitar es, como se observa, la reducción del objeto a las piezas que lo componen $\mathrm{y}$, asimismo, su reducción cuando pasa a ser considerado el producto de su relación con otros. Ahora bien, esto no implica que las relaciones con otros objetos no tengan su importancia o no afecten al objeto en cuestión. Como explica García al describir su postura filosófica y su rechazo a la posibilidad del in-itself:

every thing is either formally in its negative (in something other than a thing, in a non-thing), or objectively in another thing (in which case the comprehended thing becomes an object) [...] Each thing -whether it is my finger or my hand or a table-is a "thing" insofar as it is alone, that is, a solitary thing existing only in its negative. The table is only something, in the widest and least determinate sense, when it is comprehended in everything that is not the table. On the other hand, comprehended in the set of other tables, or in this room, or in my perception, the table becomes an object: a piece of furniture, a particular table, endowed with a color, a weight, and all that, for Harman, would emanate from its number of qualities. But the table, neither as a thing nor as an object, is not in-itself. As a thing the table is in the world, and as an object the table is in other objects that give it all its determinations and qualities. $(6)^{3}$

Si he traído a colación estos interrogantes es porque creo que ayudan a comprender mejor la dinámica compleja que el poemario de Berenguer postula como una de las claves de entrada de lectura y según la cual la identidad, tanto del sujeto humano como de la fruta, oscila entre la imposibilidad de evitar la relación con el otro (y los cambios y proyecciones que produce) así como la imposibilidad de ignorar la alteridad (en tanto aquello que no se manifiesta por completo, aquello que no se resuelve) de la fruta y del propio sujeto humano. En otras palabras, tanto los reparos de Harman a García y de García a Harman en lo que a la posibilidad del in-itself se refiere, muestran la dificutlad de negar por completo la relación o el en sí del objeto o del sujeto.

Si el primer poema, el de las varias manzanas, ponía en primer plano la dificultad de un sujeto y el del damasco intentaba dar cabida a esta posibilidad de la fruta en sí

\footnotetext{
3 Para Harman esta negación de García del in-itself es lo que acercaría su posición (la de García) a una hipersensibilidad y, en consecuencia, a una ontología relacional, de allí que cuestione esa negación: "Although Garcia initially seems to agree on this point, by making the object the difference between that which is in it and that in which it is, rather than avoiding both undermining and overmining (as I aim to do) he seems to perform both reductive operations simultaneously. For if a tree is the difference between its physical components and its current contextual position, then it will be hyper-sensitively dependent on both of these. To remove one needle from the pine tree will obviously change the "difference" between the tree's parts and its environment, and the same holds true if another nearby tree is knocked down. The only way to prevent such ontological hypersensitivity is to accept a tree-in-itself midway between the two extremes that is not overly sensitive to all changes in those extremes. But by excluding the thing-in-itself from the outset, Garcia ends up with a blatantly relational ontology, since objects will now inevitably shift whenever their inner or outer relations shift" ("Tristam García and the Thing-inItself" 6).
}

Revista Iberoamericana, Vol. LXXXII, Núm. 257, Octubre-Diciembre 2016, 135-153 
sin otro tipo de relación, quisiera analizar ahora un tercer poema que retoma los dos anteriores y que me permite iniciar, aunque no sea más que tímidamente, el paso hacia aquellos otros poemas del libro (la mayor parte) donde la relación entre el sujento que enuncia y percibe y la fruta percibida (y en su alteridad irreductible) se conjugan en un mismo espacio poético, se afectan mutuamente pero resguardando cierta alteridad. El poema en cuestión está dedicado al limón y es el más breve del libro puesto que consta tan solo de cuatro versos:

Una docena de tigres caben en un limón.

Se pueden domar los tigres:

en el limón sólo cabe

recordar el azahar. (423)

Como se observa, tampoco en este poema encontramos una clara marca de enunciación. Quién enuncia lo que se enuncia y desde dónde, no puede especificarse. No obstante, sí encontramos un claro sujeto gramatical, los tigres o, mejor, una docena de tigres, de los que por un lado se predica que pueden caber en un limón y también que pueden ser domados (aunque no se especfica por quién, ¿por un humano, por otros tigres, por su caber en el limón?). Es decir los tigres son presentados en cierto modo como activos (aunque confinados al limón en su actividad) o pasivos. Pero no son los tigres los que me interesan sino el limón en tanto objeto percibido sobre el cual se busca aprehender o delimitar la identidad.

En este caso, y una vez más Berenguer vuelve a sorprender con un giro, el limón es presentado desde una lateralidad (en sentido literal y figurado al mismo tiempo): es presentado desde su posición en el espacio y sin necesidad del verbo "ser" como en el caso de los damascos. Ahora bien, en relación con los otros poemas, aquí tampoco parece tener lugar un sujeto humano que enuncie y perciba al limón y en ese sentido se continúa el intento del poema sobre el damasco y sobre la manzana de tratar de presentar o de decir algo sobre la fruta que no implique una consciencia perceptora que la reduzca a un fenómeno de conciencia. Pero nuevamente encontramos algunos reparos, sútilmente manejados, como el uso del verbo "caber" en el primer verso y de la esctructura cabe + infinitivo, cortada en los versos tres y cuatro. Ese corte, esa incisión en el verso y en la estructura "cabe recordar" permite la aparición de la relación y al mismo tiempo su límite. El limón sólo es presentado como un receptáculo, como un lugar en el cual ocurre algo o se deposita algo o en el cual habita algo (o alguien). Pero también el que el limón sea un lugar implica una relación con aquello que habita el lugar u ocurre en el lugar. De este modo, el corte del verso, el corte de la estructura verbal puede ser leído como un reafirmar al limón en tanto lugar (de nuevo el verbo caber) pero también como una metonimia de la relación (el verbo caber relacionado, en este contexto, con el infinitivo).

Revista Iberoamericana, Vol. LXXXII, Núm. 257, Octubre-Diciembre 2016, 135-153 ISSN 0034-9631 (Impreso)

ISSN 2154-4794 (Electrónico) 
¿Pero metonimia de qué? ¿Relación con qué o de qué con qué? Por un lado, del limón con el azahar, la flor del limonero, que así como los tigres, vuelve a poner la identidad del limón en relación con otros objetos, en este caso, con una parte del árbol del que surge el propio limón (y aquí resuena la circularidad del damasco). Pero también metonimia a partir del verbo recordar que permite la entrada de un otro que recuerda, es decir, del sujeto, que establece la relación entre el limón y el azahar. Sin embargo, eso no es todo lo que la incisión nos muestra. En última instancia, lo que la incisión en la estructura cabe + infinitivo pone en primer plano (y de allí que sea una metonimia del resto del poemario) es la tensión entre la identidad relacional y ese initself que encontramos en Heidegger retomado por Harman y García en sus reparos frente a la primacía de la conciencia perceptora.

Al cortar la relación entre cabe y el infinitivo, la incisión del tercer verso pone en tensión la identidad relacional del limón, del azahar, de los tigres y de ese resto de sujeto que recuerda (en tanto tensión entre esos divesos objetos o sujetos), pero también en relación con ese in-itself tan problemático (el objeto real de Harman o la cosa-en-el-mundo de García) que no parece mostrarse del todo. Así, la irregularidad gramatical producida por el corte del verso puede ser leída como ese resto que posibilita la relación, que la estimula (en el sentido que el inglés da a "to encourage") pero también como aquello que el limón no muestra (el tercer verso si se lee "en el limón sólo cabe" produce una incapacidad, una incompletud), es decir, como aquello que oculta, como la tierra en Heidegger. De todas maneras, el recordar se cuela en la lectura, se produce el encabalgamiento y el sujeto regresa a dónde nunca se fue aunque con la certeza de la imposibilidad de agotar el objeto ni de cerrar su propio proceso de figuración. ${ }^{4}$

A esta dinámica responden, según mi parecer, la mayoría de los poemas del libro. ${ }^{5}$

4 Esta superposición de posibilidades podría verse en lo que Paul de Man dice a partir de un famoso pasaje de Proust al comienzo de Allegories of Reading donde comenta la imposibilidad de decidir en el pasaje si lo que prima es la metáfora o la metonimia: "For if we then ask the obvious and simple next question, whether the rhetorical mode of the text in question is that of metaphor or metonymy, it is impossible to give an answer. Individual metaphors, such as the chiaroscuro effect or the butterfly, are shown to be subordinate figures in a general clause whose syntax is metonymic; from this point of view, it seems that the rhetoric is superseded by a grammar that deconstructs it. But this metonymic clause has as its subject a voice whose relationship to this clause is again metaphorical. The narrator who tell us about the impossibility of metaphor is himself, or itself, a metaphor, the metaphor of grammatical syntagm whose meaning is the denial of metaphor stated, by antiphrasis, as its priority" (18). En el poemario de Berenguer la tensión, que no es necesariamente y en todos los casos entre la metáfora y la metonimia, sino entre el sujeto que enuncia y/o percibe y la fruta que percibe o que se intenta definir, no se resuelve no porque no se postule una preeminencia (de hecho la perspectiva del sujeto es la que predomina) sino porque se postulan, como veremos, la proyección del sujeto en la fruta y de la fruta en el sujeto pero también la irreductibilidad total, la asimilación total, de una parte en la otra.

5 En un texto de corte ensayístico aunque con citas de varios poemas de, principalmente, Quehaceres e invenciones (1963) encontramos algunas reflexiones de Berenguer en torno al otro y a su relación con el sujeto con el que se relaciona, sea que se trate de otros sujetos humanos o de objetos no humanos:

Revista Iberoamericana, Vol. LXXXII, Núm. 257, Octubre-Diciembre 2016, 135-153 
Tomemos como ejemplo el poema III, "(la naranja)", en el cual encontramos un sujeto que interactúa con el objeto, que se apropia de él pero que al hacerlo o para hacerlo, parece desprenderse de una parte de su cuerpo:

Yace un sol poniente

en la fuente donde brilla

la naranja del postre.

Los gajos encendidos

y la ceniza de lo oscuro

dan pasos en mi boca:

se encuentran en un bosque a medianoche

con los salteadores del placer

y una ácida aparición

y un terror que los sigue desde lejos.

Los gajos y mi lengua saben más que yo. (421)

A diferencia del grupo de poemas que he comentado hasta ahora, aquí encontramos un deíctico hacia el final y dos posesivos "mi” que remiten a una instancia de enunciación en primera personal del singular, lo cual no impide, sin embargo, que la primera estrofa del poema no involucre ninguna referencia a dicho deítico y funcione como una zona "otra" frente a la de la enunciación del sujeto. Este detalle, en apariencia nimio, cobra otro valor cuando se conecta con el espacio donde sucede la irrupción de la naranja. Más allá de la metáfora visual de la naranja como un sol de atardecer, lo que me interesa retener acá es el hecho de que la naranja adquiere, aun cuando aparezca dentro de una subordinada, una presencia diferente en tanto que naranja que brilla. Hay allí una relación entre la naranja y el brillo que difiere de la de los atributos usados para el damasco. De hecho no se trata de una naranja brillante sino de una naranja que brilla,

"Es cuando se comprueba viva, compartida y alucinadamente la presencia de los otros. Se siente, y me parece que a todos nos pasa lo mismo, la integración de nuestra propia personalidad en ese mundo colectivo, el nacimiento de fuertes corrientes de interacción. Creo que esta vivencia impersonal tan vasta de nosotros en los otros, o viceversa, es en gran parte el fundamento de toda comunicación" (100). Y agrega casi a continuación: "Pero también y simultáneamente apelamos al otro como individuo, como conciencia singular. No hay arte más que por y para otro, y así mismo del otro, es decir, desde el otro, por el otro, y para el otro. En el centro estaría el yo individual del creador como un prestidigitador atravesado de espadas" (100). También en esta línea van las apreciaciones de Amir Hamed aunque no se centra en un poemario en particular: "la voz conjuga la vida doméstica con una constante observación del mundo que la rodea, se construye a sí misma y va conquistando lugares. Su obra es perpetua re"Composicion de lugar": los referentes se convierten en su "Materia prima" a la que la escritura va reelaborando o recolocando (73).

Revista Iberoamericana, Vol. LXXXII, Núm. 257, Octubre-Diciembre 2016, 135-153 ISSN 0034-9631 (Impreso) ISSN 2154-4794 (Electrónico) 
esto es, no de un atributo impuesto a la naranja como en el caso del damasco fresco o la manzana saboreada, sino de una acción predicada de la naranja, a partir de la naranja.

La estrofa siguiente presenta una personificación de los gajos y de las cenizas. Y remarco lo de personificación porque a diferencia del brillar de la naranja anterior, que implica una relación entre un sujeto perceptor, una serie de valores donde inscribir lo brillante frente a lo no brillante, etc, y los atributos propios de la naranja, en este caso lo que se predica de los gajos y la ceniza son acciones de un sujeto humano. Así, tenemos dos tipos de relaciones, la que permite pensar la naranja que brilla (y que está implícita) y la que postula la personificación de la segunda estrofa (y que es explícita).

Ahora bien, esta segunda estrofa muestra la asimilación de la fruta con la boca del sujeto que enuncia (y que degusta la fruta) y describe las sensaciones que produce en el sujeto la naranja. Lo interesante es que en la personifiación encontramos elementos que no pueden indentificarse fácilmente como lo "oscuro" $\mathrm{y}$, principalmente, el "terror". Este último no puede ser asimilado por completo por el sujeto que se ve impedido de reducir al objeto a una percepción suya, como parecía que acontecía con las personificaciones. En otras palabras, hay una sensación de la que se puede decir que viene de lejos, pero que no se puede terminar de configurar, de asimilar y entender del todo. Esta sensación se conecta con el brillar de la naranja que excede y, por lo tanto, oculta al sujeto (que percibe el brillo de la naranja que brilla) algo de sí en tanto sujeto. Pero también excede a la naranja en tanto que tiene algo que posibilitaría el brillo (o lo que el sujeto que enuncia llama o entiende por brillo).

Y lo mismo para el caso de la sensación gustativa. El sujeto parece, por un lado, querer asimilar y estar asimilando la naranaja a lo conocido (y en este sentido la personifiación de los versos “extraña", hace extraña, una actividad común para muchos lectores, para muchos humanos, ya que permite detenerse en la sensación de comer una naranja de un modo distinto, pero lo hace a partir de la asimilación de la naranja, como si para extrañar hubiera que asimilar primero). Pero dicho sujeto debe aceptar que incluso en esa personificación hay un "terror" que sólo puede ser identificado como terror y que no logra ser identificado completamente (y por eso su carácter de uncanny). De allí entonces que el magistral verso final "extrañe" y en cierto modo escinda al propio sujeto que debe aceptar eso de irreductible que hay, si no en la naranja en sí, al menos en el saborear la naranja.

Así entonces vemos en un mismo poema la relación entre el sujeto que enuncia y que come con la naranja percibida y que es comida pero también un límite a la sensación que es un límite a la posibilidad del sujeto de figurase como una entidad plenamente consciente y coherente y totalmente asimilada. Algo, algo como el terror que viene de lejos o el "que brilla" de la naranja, irrumpe en la escena y no permite, aun cuando el sujeto devore a la naranja, que aquel asimile la identidad de esta a su percepción, que la relación se constituya en lo único existente, ni que el sujeto se des-cubra por

Revista Iberoamericana, Vol. LXXXII, Núm. 257, Octubre-Diciembre 2016, 135-153 ISSN 0034-9631 (Impreso)

ISSN 2154-4794 (Electrónico) 
completo. Por el contrario lo que parece des-cubrir el sujeto es esa zona otra, oculta de la naranja y de sí mismo que se repliega constantemente.

Algo similar encontramos en el poema dedicado al durazno:

Me gusta el durazno maduro:

me gusta el área de perfumes

el ruburoso terciopelo

que rodea

su jardín particular.

No veo el carozo escondido

ese crustáceo amargo

en la cueva de pulpa

rosamarilla.

Sé que tropezaré con él

y quizás me salte un viejo diente de leche.

En el plato (asustadas como rocío)

han quedado unas gotas:

¿almíbar?

¿sangre?

En este caso el sujeto se adueña del comienzo y deja para el final la escena de un plato con unas gotas, en contraposición al poema de la naranja donde se parte del plato con la naranaja intacta hacia el sujeto escindido. Pero el resultado, con matices, es, sin embargo, parecido. El sujeto trata de asimilar la forma y la identidad del durazno a lo conocido (perfumes, terciopelo, jardín) aunque sin llegar a una personificación como en el caso anterior. Debe reconocer que no ve el carozo, que acecha en lo profundo, pero eso no impide que una experiencia anterior le permita afirmar que se topará con él, que tropezará con él. Inclusive esa particularidad del durazno, esa interioridad, parece manejable. Sin embargo, la presencia de las gotas asustadas (¿se trata de una traslación? ¿No será más bien que el sujeto está asustado?) vuelve a subrayar la imposibilidad de negar la importancia de la relación para la conformación de la identidad de la fruta y del sujeto (ambas se afectan mutuamente y en esta caso específico en sus cuerpos) pero también su irreductibilidad a esa relación. De hecho, la sangre que se menciona podría leerse más allá de la fractura hipotética de un viejo diente de leche y pensarse más bien como otra manifestación más violenta, concreta y explícita, del terror evocado al comer la naranja. Es el recuerdo de la imposibilidad de negar la relación entre el sujeto y el durazno pero también del des-cubrimiento total de la identidad de la fruta y del sujeto que no puede manejar por completo su cuerpo (el hipotético diente de leche), lo que parece asomar en esas gotas de almíbar o sangre.

Quisera, para concluir, analizar dos últimos poemas (aunque se podrían mencionar unos cuantos como el primero sobre la manzana o el de las uvas). He dejado fuera de

Revista Iberoamericana, Vol. LXXXII, Núm. 257, Octubre-Diciembre 2016, 135-153 ISSN 0034-9631 (Impreso)

ISSN 2154-4794 (Electrónico) 
manera consciente aquellos poemas en los que junto a la dialéctica de un sujeto que enuncia y percibe una fruta y una fruta que se le planta como un límite, encontramos referencias a lugares precisos como Montevideo (en el caso del poema sobre "(las almendras)") o diversos biografemas como en el caso del poema dedicado al ananá o en el poema final dedicado al maní y en el que apreciamos la referencia a un sujeto de la infancia. Si los he dejado afuera es porque plantean una cuestión que excede el objetivo, siempre parcial, de este ensayo: la de la autofiguración de corte biográfica. De todos modos, aun en esos poemas, la tensión que hemos visto moldea la descripción de la identidad de la fruta, no desaparece sino que se agudiza y se inscribe en la dinámica autobiográfica.

Hecha la salvedad quisiera pasar entonces al primero de estos dos poemas, aquel dedicado a la sandía:

Yo buscaba sin saber bien
qué era repartir aquella extensa fruta.
Repartir la sandía -me dije--
y sacrificamos en tajadas
su fresca encarnadura.

Quedó abierta sobre la mesa mostrando el corazón. ¿De la tarde? ¿De la casa? ¿Del silencio?

Repartir la sandía - me dijees repartir una siesta de verano una estación con vidrieras rojas y desierta una cueva verde habitada por la sed. (439)

En este poema el deíctico de enunciación de primera persona es lo primero que irrumpe. Su fuerza es tal que anticipa la de las tajadas con las que un yo plural oficiará el sacrificio de la sandía (y hay que entender el genitivo como la acción sobre la sandía pero tambien sobre un tipo de sacrificio que inaugura la sandía, que le es, en cierta medida, propio). Pero si bien esa fuerza diferencia el poema de los otros que he mencionado hasta el momento, en los cuales podía, o no, haber un yo en función de enunciante y perceptor (aunque nunca en esa posición inicial tan destacada en el verso), en seguida esa centralidad se ve matizada por la duda de una búqueda cuyo cometido es, en última instancia, repartir aquella extensa fruta, la sandía.

Nótese, sin embargo, un detalle. Lo que motiva la duda del sujeto lírico no es la posibilidad de asimilar la identidad de la fruta sino la ignorancia del ritual apto para repartirla. Pareciera, en una primera instancia, que, a diferencia de otros poemas, el sujeto teme afrontar solo o sola la presencia de la sandía y de allí que desplace la duda

Revista Iberoamericana, Vol. LXXXII, Núm. 257, Octubre-Diciembre 2016, 135-153 ISSN 0034-9631 (Impreso) 
sobre la posibilidad de perfilar la identidad de la sandía al modo de repartición. Esto se confirma en la necesidad de que el sacrifico se produzca, como ya anticipé, en compañía de otros/as y como condición previa a toda descripción de la sandía.

Ahora bien, una vez iniciado el ritual, los temores ante la incapacidad de la búsqueda, ante la incapacidad de no lograr saber cómo repartir la sandía (en última instancia cómo asimilarla a lo conocido y manipulable), no desaparecen, se acentúan. La presencia de la duda, la presencia de la inquitante sandía y de la dificultad para repartirla ha sido mitigada mediante las tajadas; pero ese corte, como aquella incisión del verso en el poema sobre el limón y que separaba el "cabe" del "recordar", vuelve a poner en primer plano la relación del sujeto con la sandía y su posibilidad o imposibilidad de alcanzar esa identidad y en última instancia la suya propia. La sandía se erige entonces, aunque no es fácil percibirlo, en un horizonte de posibles respuestas (y temores) pero también en un límite. ${ }^{6}$

Dije antes que en esta relación (¿dialéctica?) entre el sujeto y el objeto, en este caso la fruta, ambos mostraban algo, proyectaban un mundo (para retomar a Heidegger) pero sin mostralo (del) todo, replegando algo. ¿Cómo acontece eso acá? ¿Cómo acontece esto en un poema en el cual poco se dice sobre la sandía previa al sacrificio y donde el final del poema es una enumeración de cosas y evocaciones que remiten no a la sandía si no al repartir la sandía?

Lo primero que hay que señalar es la presencia del infinitivo "repartir" en tanto acción no conjugada verbalmente que se repite en varios versos y que contrasta con otros verbos conjugados en pasado como "dije" "buscaba", "quedó", "era" y "sacrificamos". Todos estos verbos en pasado indican el momento de dudas incial del yo y el sacrificio de la sandía, pero la repartición está en infinitivo y en cierto modo transciende el sacrificio particular ya sea porque lo inscribe en una repetición ritual (que se actualiza cada vez que se rebana una sandía y se comparte), ya sea porque lo que se reparte y está en juego no es sólo un pedazo de la sandía sino aquello que no se puede asimilar por completo.

El repartir la sandía, el dejarla sobre la mesa con el corazón al desnudo, no implica, de parte del sujeto lírico, el haber asimilado o captado la identidad de la sandía o el haber cerrado toda relación para marcar una distancia total en la que el sujeto utiliza la sandía sin consecuencias (para su propio ser sujeto). Lo que el repartir permite es

${ }^{6} \mathrm{Al}$ respecto valgan las palabras de Michel Collot: "Grace à autrui j'accède à l'infini, mais je découvre aussi ma finitude. L'autre introduit dans les limites de mon champ visuel une infinité de points de vue possibles, mais il demeure pour sa part impossible à voir. Sur ce point, Lévinas et Blanchot ont parfaitement raison: autrui échappe à mon regard. Mais cette invisibilité absolue ne saurait absoudre autrui de l'ordre du visible, puisqu'elle en est le fondement même; et loin de faire de l'Autre un 'homme sans horizon', c'est elle qui inscrit dans le monde la structure d'horizon, et qui fait d'autrui lui-même un indépassable horizon" (94).

Revista Iberoamericana, Vol. LXXXII, Núm. 257, Octubre-Diciembre 2016, 135-153 ISSN 0034-9631 (Impreso)

ISSN 2154-4794 (Electrónico) 
reactualizar la relación del sujeto con la sandía la cual pasa a ser un límite y un horizonte, para retomar a Collot, aquello que no se muestra ni siquiera tras su destrucción y que impide al sujeto clausurar la duda inicial, y, por ende, trasladarla a las preguntas del séptimo verso o de las evocaciones finales.

Sin embargo, al abrir y posibilitar las evocaciones, el repartir le recuerda al sujeto que hay algo en la fruta (y por proyección en su identidad) que no puede asimilarse por completo y de una manera coherente. Dicho de otro modo, si el sujeto ha logrado, en compañía de otros, repartir la sandía, ha debido aceptar (y eso manifiestan las preguntas sobre el corazón de la sandía y las evocaciones carentes de tiempo del final) que esa repartición no ha logrado incorporarse al paso del tiempo, no ha logrado conjugarse en un tiempo verbal que la deje atrás y permita borrar la duda. Esa duda inicial sobre cómo repartir la sandía se continúa, se reactualiza (porque en definitiva siempre estuvo latente), pero ahora desde un sujeto que pone su identidad en relación a y en merced de todo aquello que la sandía como horizonte evoca pero también aquello que no muestra, aquello que no se asimila al tiempo ni a la percepción y que queda englobado en la repetición de un infinitivo y en la postulación de la sed que lejos de saciarse, al acceder al corazón de la fruta, se intensifica aun en el propio sujeto que des-cubre sus propios límites en tanto tal.

Por último, quisiera detenerme en el poema dedicado al cacao, el único de todo el libro en el que se apostrofa a la fruta: ${ }^{7}$

Querido:

de tí en esta 35 latitud Sur

no conozco más que tu investidura

la apasionada condecoración de tu manteca

tu azúcar

tu vino

y tu ardiente melancolía.

El Chocolate te ha nombrado

de una sola vez

definitiva

y entonces he sabido quien eres

como si hubieran cantado en mi boca

oscuros mirlos

enamorados.

No necesito descifrar el canto.

Quiero solo tus mirlos. (469)

7 Y recordemos la reversibilidad que Culler (aunque no es el único obviamente) ha destacado como una de las características más importantes del apóstrofe en su relación con el yo lírico (Véase "Apostrophe”).

Revista Iberoamericana, Vol. LXXXII, Núm. 257, Octubre-Diciembre 2016, 135-153 ISSN 0034-9631 (Impreso)

ISSN 2154-4794 (Electrónico) 
Además de ser el penúltimo poema del libro (el último es uno de los de corte autofigurativo), este texto dedicado al cacao es uno de los pocos en los que el sujeto lírico pone de manifiesto cierta actitud celebratoria en relación con la (im)posibilidad de acceder a la fruta en cuestión y a su propia identidad. Si ya el poema de la sandía anticipaba el ritual en tanto que actividad colectiva aquí parece descartarse su carácter de fiesta. La renuncia con que se cierra el poema no impide la manifestación del deseo (que de hecho la sigue) ni pretende ser una contradicción; por el contrario, dicha renuncia implica una posibilidad, supone el deseo y lo potencia pero siempre a raya, sin dejar que se actualice por completo y pierda su fuerza.

En este sentido no es llamativa la irrupción del chocolate como aquel elemento encargado de definir al cacao, ya que es ese nombrar (e insisto en la importancia del apóstrofe del verso inicial, único en el libro) el que resume la tensión que he tratado de destacar entre el sujeto y el objeto. Si el cacao sólo puede conocerse, en el caso puntual del sujeto que enuncia, a partir de sus manifestaciones (de las apasionadas condecoraciones, para ser más precisos), el sujeto no ha logrado aprehender dicha identidad en su totalidad, en su cosa en sí, puesto que algo del cacao permanece (¿irremediablemente? ¿afortunadamente?) escondido en el chocolate y en el hipotético canto de los hipotéticos y oscuros mirlos, así como también algo de hipotético y oculto hay en el canto, en el poema.

Ahora bien, lejos de renunciar al cacao, lejos de renunciar al canto y, por metonimia, a su propia identidad de yo poeta, el sujeto lírico se conforma con la incomprensión, con la oscuridad del canto de los mirlos que no precisa ser descifrado del todo y que aún permite la relación del yo con el cacao, del cacao con sus manifestaciones y del yo con su canto. Así, entonces, el poema se presenta como un contrapunto del poema del damasco y como una prolongación del de la sandía, es decir, como un poema circular en cierto modo pero ya no en tanto circularidad como un volverse en sí de la fruta sino más bien como un volverse en sí del canto en tanto que chocolate, en tanto que condecoración apasionada que rechaza voluntariamente la posibilidad de alcanzar la identidad de la fruta para acentuar su relación con ella y con su propia actividad poética en un clima que además de reflexivo es, al menos en este poema puntual, festivo.

He intentado en estas páginas desentrañar uno de los aspectos más complejos y recurrentes de una obra tan diversa y versátil como la de Amanda Berenguer en la cual el sujeto lírico no desaparece pese a que se trata de una de las poéticas donde la presencia del otro (en sus múltiples caras) es tan desafiante y ambigua. En el caso de Identidad de ciertas frutas he intentado mostrar cómo opera esa tensión entre el sujeto y los objetos, las frutas, que lejos de resolverse se reactuliza con diversas variantes en cada poema en particular. Aun así, y pese a la variedad de formas métricas y de abordajes que el poemario muestra, creo que puede apreciarse una constante necesidad, en gran parte de estos textos, de poner en relación al sujeto con la fruta y con otros objetos (y a la

Revista Iberoamericana, Vol. LXXXII, Núm. 257, Octubre-Diciembre 2016, 135-153 ISSN 0034-9631 (Impreso) 
fruta con esos otros objetos). Y esto con el fin de repensar y de rearticular la noción de identidad a partir de una dinámica que no se resuelva en la desaparición total del sujeto y el objeto en la relación ni en una alteridad infranqueable, ni en la fruta como una mera proyección. Se trata más bien de apreciar que al mismo tiempo que la relación abre y proyecta nuevos horizontes, la presencia del otro y del resto (en tanto que otro y del sí-mismo-como-otro que esa presencia inagura) les impone un límite al sujeto y al objeto que, paradojicamente, des-cubren y posibilitan un des-cubrimiento siempre parcial y en constante tensión.

\section{OBRAS CITADAS}

Berenguer, Amanda. "Identidad de ciertas frutas". [1983]. Constelación del navío. Poesía 1950-2002. Montevideo: H editores, 2002.

El monstruo incesante. (Expedición de caza). Montevideo: Arca, 1990.

Collot, Michel. La poésie moderne et la structure d'horizon. Paris: Puf, 1989.

Culler, Jonathan. “Apostrophe.” Diacritics 7/4 (1977): 59-69.

La poética estructuralista El estructuralismo, la lingüistica y el estudio de la literatura. [1975]. Barcelona: Anagrama, 1978.

de Man, Paul. Allegories of Reading. Figural Language in Rousseau, Nietzsche, Rilke, and Proust. New Haven: Yale UP, 1979.

García, Tristan. "Crossing Ways of Thinking: On Graham Harman's System and my Own." Mark Allan Ohm, trad. Parrhesia 16 (2013): 14-25.

Harman, Graham. "Object-Oriented France: The Philosophy of Tristan García." Continent 5/1 (2012): 6-21.

The Quadruple Object. London: Zero Books, 2011.

"Tristan García and the Thing-in-itself." Parrhesia 16 (2013): 26-34.

Heidegger, Martin. "El origen de la obra de arte". Caminos de bosque. Helena Cortés y Arturo Leyte, trads. Madrid: Alianza, 2010.

Irigoyen, Emilio. "Tridimensionalidad de la palabra y placer (¿femenino?) de la escritura en la obra de Amanda Berenguer". Berenguer, Amanda. La estranguladora. Montevideo: Cal y canto, 1998.

Jitrik, Noé. "Poética y referencialidad". Línea de flotación. Roberto Ferro, ed. Buenos Aires: El otro, el mismo, 2002. 
